\title{
Metabolic Shift in Cancer Cells Treated with Chemotherapy: Autophagy and Cancer Chemoresistance
}

\section{Crispin R. Dass*}

School of Biomedical and Health Sciences, Victoria University, St Albans 3021, Australia

Keywords: Metabolism; Autophagy; Cancer; Chemotherapy

\section{Editorial}

Chemotherapy is the use of chemical drugs to destroy neoplastic cells. Consisting of a cocktail of drugs, chemotherapy is used either on its own or in combination with surgery or radiation to kill or eradicate as many cancer cells as possible. It is common knowledge now that most drugs harbour significant side-effects plus the cancer develops resistance to the drug. A relatively new hypothesis in cancer therapy, proven to some extent, is the cell biological phenomenon of autophagy, the process by which the cell degrades its own cytoplasmic components and organelles. This catabolic process is important in maintaining some form of cellular homeostasis when cells are faced with nutrient deficiencies and other cellular stresses. Autophagy prevents damage that can occur via the accumulation of protein aggregates, hypoxic environments and dysfunctional organelles through degradation and nutrient production, and can in fact prove to be beneficial in such pathologies as Alzheimer's disease.

Autophagy is instrumental in providing energy for metabolism and thus meeting the increased energy demands of cancer cells, to enable them to grow and proliferate. For instance, it has been reported that loss of stromal caveolin-1 (Cav-1) is an indicator of poor prognosis in cancer [1]. It has been proposed that fibroblasts are targeted by neighbouring cancer cells, with the latter invoking oxidative stress on the former [2]. As a result, autophagy is induced via two protein inducers - HIFla (hypoxia-inducible factor 1a) and NFkB (nuclear factor $\mathrm{kB}$ ). This causes the degradation of Cav-1 and mitochondria through an increase in the level of key regulators - BNIP3 (Bcl-2/ adenovirus E1B $19 \mathrm{kDa}$ protein-interacting protein 3) and BNIP3L (Bcl2/adenovirus $\mathrm{E} 1 \mathrm{~B} 19 \mathrm{kDa}$ protein-interacting protein 3-like), forcing a change in metabolism to aerobic glycolysis, where lactate, ketones and glutamine byproducts are synthesized. In almost a parasitic fashion, these byproducts are taken up by neighbouring cancer cells which use them to fuel production of ATP (adenosine triphosphate) to meet their survival demands.

A similar case is active uptake of glutamine, produced by fibroblasts, by surrounding cancer cells. Glutamine is reduced to ammonia during oxidative metabolism and ammonia directly induces autophagy [3]. Thus, the continual induction of autophagy by cancer cells is a means by which they can survive adverse nutritional states. Finally, when melanoma cell lines are deprived of leucine, apoptosis is activated [4]. Although the activation of autophagy is decreased, mTORC1 is not inhibited (as has been noted in other amino acid deprivation models). There was an increase in apoptosis when leucine deprivation was combined with autophagic inhibitors. Interestingly, in leucine-deprived melanoma cell lines treated with mTORC1 inhibitor, a reactivation of autophagy was noted.

Thus, the link between cancer cells and metabolic pathways is further extended with the finding that autophagy allows nutritionallystressed cancer cells to survive and even overcome drug toxicity [5]. This leads to reduced chemotherapy response in patients, and therefore, lack of proper tumour management and the associated low quality of life and economic burden to the patient, his/her loved ones and the healthcare system in general. Cancer still largely remains an incurable pathology, and it is hoped that a better understanding of the molecular mechanisms of autophagy and how it 'empowers' tumour cells will lead to better treatment regimens in future.

\section{References}

1. Witkiewicz AK, Dasgupta A, Sotgia F, Mercier I, Pestell RG, et al. (2009) An absence of stromal caveolin-1 expression predicts early tumor recurrence and poor clinical outcome in human breast cancers. Am J Pathol 174: 2023-2034.

2. Lisanti MP, Martinez-Outschoorn UE, Pavlides S, Whitaker-Menezes D, Pestel RG, et al. (2011)Accelerated aging in the tumor microenvironment: connecting aging, inflammation and cancer metabolism with personalized medicine. Cell Cycle 10: 2059-2063.

3. Ko YH, Lin Z, Flomenberg N, Pestell RG, Howell A, et al. (2011) Glutamine fuels a vicious cycle of autophagy in the tumor stroma and oxidative mitochondria metabolism in epithelial cancer cells: Implications for preventing chemotherapy resistance. Cancer Biol Ther 12.

4. Sheen JH, Zoncu R, Kim D, Sabatini DM (2011) Defective regulation of autophagy upon leucine deprivation reveals a targetable liability of human melanoma cells in vitro and in vivo. Cancer Cell 19: 613-628.

5. Tan ML, Friedhuber AM, Dunstan DE, Choong PF, Dass CR (2010) The performance of doxorubicin encapsulated in chitosan-dextran sulphate microparticles in an osteosarcoma model. Biomaterials 31: 541-551.

*Corresponding author: Crispin R. Dass, School of Biomedical and Health Sciences, Victoria University, St Albans 3021, Australia, Tel: +61 399192419 , Fax: +61 39416 2465; E-mail: crispin.dass@vu.edu.au

Received October 20, 2012; Accepted October 22, 2012; Published October 29, 2012

Citation: Dass CR (2013) Metabolic Shift in Cancer Cells Treated with Chemotherapy: Autophagy and Cancer Chemoresistance. Metabolomics 3: e123. doi:10.4172/2153-0769.1000e123

Copyright: (C) 2013 Dass CR. This is an open-access article distributed under the terms of the Creative Commons Attribution License, which permits unrestricted use, distribution, and reproduction in any medium, provided the original author and source are credited. 